\title{
Predicting patient outcomes in psychiatric hospitals with routine data: a machine learning approach
}

\author{
J. Wolff ${ }^{1,2^{*}}$ (D) A. Gary ${ }^{3}$, D. Jung ${ }^{4}$, C. Normann ${ }^{1}$, K. Kaier ${ }^{5}$, H. Binder ${ }^{5}$, K. Domschke ${ }^{1}$, A. Klimke ${ }^{6,7}$ and M. Franz ${ }^{8,9}$
}

\begin{abstract}
Background: A common problem in machine learning applications is availability of data at the point of decision making. The aim of the present study was to use routine data readily available at admission to predict aspects relevant to the organization of psychiatric hospital care. A further aim was to compare the results of a machine learning approach with those obtained through a traditional method and those obtained through a naive baseline classifier.

Methods: The study included consecutively discharged patients between 1st of January 2017 and 31st of December 2018 from nine psychiatric hospitals in Hesse, Germany. We compared the predictive performance achieved by stochastic gradient boosting (GBM) with multiple logistic regression and a naive baseline classifier. We tested the performance of our final models on unseen patients from another calendar year and from different hospitals.

Results: The study included 45,388 inpatient episodes. The models' performance, as measured by the area under the Receiver Operating Characteristic curve, varied strongly between the predicted outcomes, with relatively high performance in the prediction of coercive treatment (area under the curve: 0.83) and 1:1 observations (0.80) and relatively poor performance in the prediction of short length of stay (0.69) and non-response to treatment (0.65). The GBM performed slightly better than logistic regression. Both approaches were substantially better than a naive prediction based solely on basic diagnostic grouping.

Conclusion: The present study has shown that administrative routine data can be used to predict aspects relevant to the organisation of psychiatric hospital care. Future research should investigate the predictive performance that is necessary to provide effective assistance in clinical practice for the benefit of both staff and patients.
\end{abstract}

Keywords: Psychiatry, Hospitals, Decision support techniques, Machine learning, Health services administration

\section{Introduction}

The individual needs of patients are central to decision making in hospital care [1]. Nevertheless, reducing complexity of individual episodes through the identification of common patterns of needs facilitates an efficient organisation of care [2].

\footnotetext{
* Correspondence: jan.wolff@uniklinik-freiburg.de

${ }^{1}$ Department of Psychiatry and Psychotherapy, Medical Center - University of Freiburg, Faculty of Medicine, University of Freiburg, Freiburg, Germany

${ }^{2}$ Department of Business Development, Evangelical Foundation Neuerkerode, Braunschweig, Germany

Full list of author information is available at the end of the article
}

The identification of common patterns of needs and the prediction of relevant aspects of patient care were found to be more complex in hospital psychiatry than in other medical disciplines [3-5]. Reasons put forward for this were less distinct diagnostic concepts [6-8], less standardisation of care [9] and a broader spectrum of acceptable therapeutic regimes [10].

Machine learning is a potent approach to identify and quantify multidimensional patterns in patient and hospital service data [11]. It has gained increasing attention in health care by achieving impressive results, for instance, in early prediction and diagnosis of breast cancer [12], acute kidney injury [13], skin cancer [14], prostate 
cancer [15], diabetic retinopathy [16] and depression [17]. Other studies applied machine learning to aspects relevant to the organisation of hospital care, such as predicting patient volume in emergency departments [1820], the management of acute sepsis [21-23] and the daily costs per psychiatric inpatient [24].

The actual use of machine learning applications in routine clinical care often lags behind prominent achievements in research projects. Most published clinical prediction models are never used in clinical practice [25]. A common problem is the availability of useful data at the point of decision making $[26,27]$.

Previous research has often included a broad set of medical, psychometric and sociodemographic variables of which many should usually not be available at admission of patients at many hospitals [3, 28]. High administrative workload in clinical staff and overall time constraints are prevalent in many health care systems [29]. Therefore, required feature variables should be routinely available at the point of decision making without further curation.

There is currently a lack of evidence informing the performance and usefulness of machine learning applications based on routine data [30]. Our study addresses this lack of evidence by restricting predictive modelling to a set of routinely available feature variables.

The aim of the present study was to use routine data readily available at admission to predict aspects relevant to the organization of psychiatric hospital care. A further aim was to compare the results of a machine learning approach with those obtained through a traditional method and those obtained through a naive baseline classifier.

\section{Methods}

The present study included all inpatient episodes that were admitted to one of nine psychiatric hospitals in Hesse, Germany, and that were discharged between 1st of January 2017 and 31st of December 2018. An inpatient episode was defined as a patient's stay at the hospital between admission and formal discharge. We excluded patients that were not in the billing class of adult psychiatry of the German lump-sum payment system for psychiatric hospital care, such as child and adolescent psychiatry and patients with mainly psychosomatic ailments. Missing data in outcome variables was addressed with listwise deletion. The study was approved by the ethics commission of the Medical Council Hesse, record number FF116/2017.

Three different modelling approaches were compared: The chosen machine learning approach was a stochastic gradient boosting algorithm implemented in the CARET package in $\mathrm{R}$ based on the gradient boosting machine (GBM) by Friedman [31-33], The traditional method was logistic regression with the full set of feature variables used in the machine learning approach. The naive baseline classifier was obtained by using only basic diagnostic groups in a logistic regression. The basic diagnostic groups were F0/ G3 Organic mental disorders, F1 Substance-related mental disorders, F2 Schizophrenia, schizotypal and delusional disorders, F3 Affective Disorders and Others.

The required data were obtained from routinely documented information in the electronic medical records and patient administration databases. A restricted set of feature variables was used that should be available in most hospitals at admission of patients. These were 1 . the one-dimensional Global Assessment of Functioning Scale (GAF) [34], 2. age, 3. gender, 4. mode and time of admission and 5. a basic diagnostic grouping (F0/G3 Organic mental disorders, F1 Substance-related mental disorders, F2 Schizophrenia, schizotypal and delusional disorders, F3 Affective Disorders and Others).

We used these features to predict the probability of 1 . non-response to therapy as defined by failing to reach the next ten-point-interval of the GAF-scale at discharge (e.g. from 21 to 28 was considered as non-response and from 28 to 31 was considered as response), 2. the need for coercive treatment, 3 . the need for 1:1 observation, 4 . the need for crisis intervention, 5. long length of stay (LOS) above the 85th percentile and 6. short LOS below the 15th percentile.

We divided data into a training set, i.e. patients discharged in the first three quarters of 2017, a validation set, i.e. patients discharged in the last quarter of 2017 and a test set, i.e. patients discharged in 2018. We engineered features and tuned hyperparameters on the basis of the trained models' performance in the validation data set. The continuous features, i.e. the GAF sore at admission and patients' age at admission, were standardised to a mean of zero and a standard deviation of one by subtracting the mean of respective variables from each value and dividing the results by the respective standard deviation. The hyperparameter tuning was carried out using the built-in tuning process in the Caret package, modifying each of the four tuning parameters, i.e. boosting iterations, max depth of trees, shrinkage and minimal terminal node size, until a maximum performance was reached in the validation sample. The performance of the final models was assessed in the held-out test data (patients discharged in 2018) to assess performance in future episodes. Thereby, we had trained nine different models, each holding-out one study-site, and used these models to predict the outcomes of patients from the held-out study site to restrict assessment of performance to hospitals not involved in the training process.

We used the area under the Receiver Operating Characteristic curve (ROC) and Precision and Recall plots (PR-Plots) to compare predictive performance. We calculated 95\% DeLong confidence intervals for the area 
under the ROC [35]. Furthermore, we defined different cut-off values for the operationalisation of the models that maximised sensitivity at a minimum precision of $0.2,0.25$ and 0.33 , representing 4,3 , and 2 false positives for each true positive prediction, respectively. We chose a threshold of 0.2 to be the minimum for a clinically meaningful application based on previous work of Tomašev et al. [13]. Furthermore, we defined a sensitivity of 0.2 as the minimum threshold for clinically meaningful application.

\section{Results}

The study included 45.388 inpatient episodes. After addressing missing data in outcome variables with listwise deletion, 40.614 episodes were included in further analyses $(89.5 \%)$. There were no missing data in feature variables after this step. Table 1 shows the characteristics of included episodes.

Table 1 Characteristics of inpatient episodes

\begin{tabular}{|c|c|c|c|c|}
\hline & 2017 & & 2018 & \\
\hline Number of Episodes (n) & 20,283 & & 20,331 & \\
\hline Age (years, mean \& SD) & 48 & 19 & 48 & 19 \\
\hline Female (n \& \%) & 8872 & 44 & 8869 & 44 \\
\hline GAF Admission (mean \& SD) & 35 & 12 & 35 & 12 \\
\hline Length of Stay (days, median \& IQR) & 16 & $8-29$ & 16 & $8-29$ \\
\hline \multicolumn{5}{|l|}{ Basic Diagnostic Grouping (n \& \%) } \\
\hline F0/G3 & 2044 & 10.1 & 2099 & 10.3 \\
\hline F1 & 7485 & 36.9 & 7649 & 37.6 \\
\hline F2 & 2929 & 14.4 & 3047 & 15.0 \\
\hline F3 & 5566 & 27.4 & 5365 & 26.4 \\
\hline Others & 2259 & 11.1 & 2171 & 10.7 \\
\hline \multicolumn{5}{|l|}{ Study site (n \& \%) } \\
\hline Site 1 & 3564 & 17.6 & 3716 & 18.3 \\
\hline Site 2 & 1313 & 6.5 & 1502 & 7.4 \\
\hline Site 3 & 2436 & 12.0 & 2548 & 12.5 \\
\hline Site 4 & 2115 & 10.4 & 1983 & 9.8 \\
\hline Site 5 & 2159 & 10.6 & 2284 & 11.2 \\
\hline Site 6 & 3854 & 19.0 & 3656 & 18.0 \\
\hline Site 7 & 1493 & 7.4 & 1446 & 7.1 \\
\hline Site 8 & 1636 & 8.1 & 1662 & 8.2 \\
\hline Site 9 & 1713 & 8.4 & 1534 & 7.5 \\
\hline 1:1 Observation ( $n$ \& \%) & 265 & 1.3 & 265 & 1.3 \\
\hline Crisis Intervention (n \& \%) & 219 & 1.1 & 192 & 0.9 \\
\hline Non-Response (n \& \%) & 5108 & 25.2 & 4617 & 22.7 \\
\hline Coercive Treatment ( $\mathrm{n} \& \%$ ) & 1306 & 6.9 & 1382 & 6.8 \\
\hline
\end{tabular}

SD Standard deviation, GAF Global Assessment of Functioning, IQR Interquartile range, F0/G3 Organic mental disorders, F1 Substance-related mental disorders, F2 Schizophrenia, schizotypal and delusional disorders, F3 Affective Disorders
Figure 1 compares the possible combinations of sensitivity, i.e. the proportion of correctly predicted actual positives, and specificity, i.e. the proportion of correctly predicted actual negatives, that were reached by the different classifications. The area under the curve is provided for each outcome and classification and 95\% confidence intervals were estimated. Furthermore, Fig. 1 shows the operational points at the curves that maximize sensitivity at a minimum precision of $0.2,0.25$ and 0.33 , respectively. Measured by the area under the curve, the models for coercive treatment, 1:1 observation, long LOS and crisis intervention achieved a relatively good performance between 0.83 and 0.74 .

Figure 2 compares the possible combinations of recall, a synonym for sensitivity, and precision, i.e. the proportion of actual positives among all positive predictions. Despite a relatively high area under the curve in Fig. 1, the models for the outcomes 1:1 observation and crisis intervention showed a poor performance in the comparison of precision and recall without clinically meaningful combinations. Table 2 provides additional measures of classification performance for the remaining outcomes based on the potentially meaningful operational points.

As mentioned above, we trained each final model on patients discharged in 2017, leaving out one site in each training round, and evaluated each model's predictive performance in patients discharged in 2018 only from the study site not included in the training. Figure 3 shows the differences in predictive performance measured by the area under the curve between the study sites. The models for coercive treatment, long LOS, short LOS and non-response to treatment showed relatively low variance in predictive performance. The models for crisis intervention and 1:1 observation performed very well in some study sites and very close to pure random classification, or worse, in others.

Figure 4 shows the top ten feature variables ordered by their importance in predicting the outcome variables in the GBM model. Variable importance is a dimensionless measure that represents the influence of each feature on the predictive performance relative to the other variables (the method is described in detail in 31). GAF at admission, age at admission and a basic diagnostic grouping at admission were important variables in most outcomes.

\section{Discussion \\ Key findings}

A common problem in the application of machine learning is availability of data at the point of decision making. The present study aimed at using routine data readily available at admission to predict aspects relevant to the organisation of psychiatric hospital care. A further aim was to compare the results of a machine learning approach to those obtained using a traditional method and those obtained using a naive baseline classifier. 


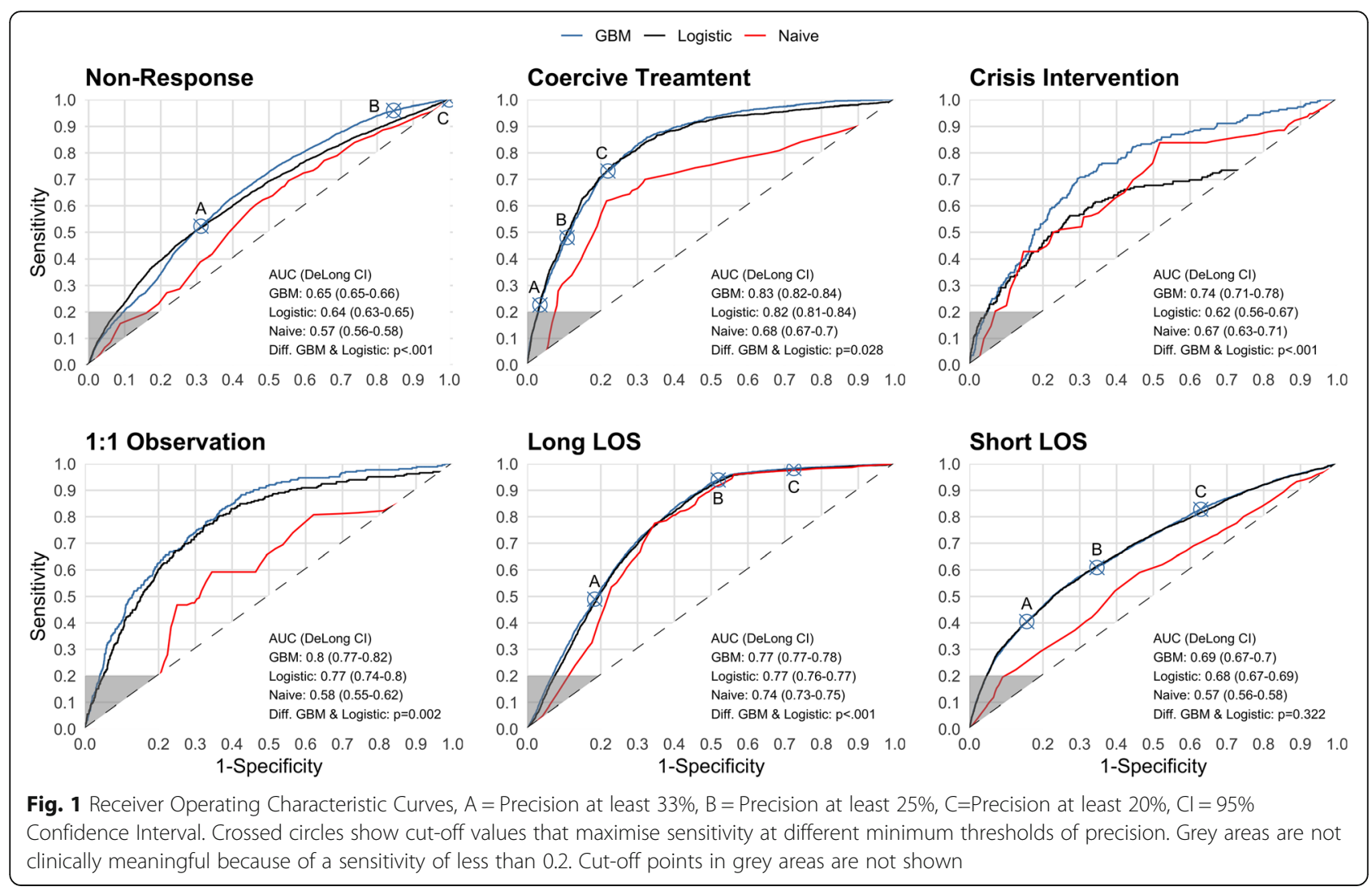

The models' performance, as measured by the area under the ROC, varied strongly between the predicted outcomes, with relatively high performance in the prediction of coercive treatment and 1:1 observations and relatively poor performance in the prediction of short LOS and non-response to treatment. The GBM performed slightly better than logistic regression. Both approaches were substantially better than a naive prediction based solely on basic diagnostic grouping.

The present results confirm previous studies suggesting inadequacy of the area under the ROC as a measure for predictive performance in unbalanced data, in our case data with many more negatives than positives [36]. The area under the ROC gave a misleadingly positive impression of the models for 1:1 observation and crisis intervention, while the precision and recall plots revealed a lack of sufficient precision for a clinically meaningful application.

Furthermore, we found relatively large differences in the areas under the ROCs between different hospital sites (Fig. 3). As described in the methods section, we trained each model in 8 hospital and used it to predict outcomes of patients from the left-out ninth hospital. Therefore, if the remaining ninth hospital had very different patients or provided care in a different way, the model would perform worse in this hospital than in the other hospitals that were more common to each other. This was probably the reason for the very low performance of the naive baseline classifier in predicting 1:1-observations in two study sites with areas under the curves below 0.5 ( 0.4 and 0.1 , respectively), where the incidences of this event were very low $(0.3$ and $0.07 \%)$.

It is still unclear, which predictive performance is sufficient for beneficial application in routine clinical practice and this was out of the scope of the present study [37, 38]. Furthermore, different clinical applications might require their own trade-off decisions between reducing false alerts and increasing coverage of actual positives. We have chosen different configurations for comparison of model performance, borrowing from Tomašev et al. in their prediction of acute kidney injuries [13]. For instance, our GBM model for the prediction of coercive treatment, operationalised with a precision of at least 0.2 (see Table 2), gave a warning for $26 \%$ of all episodes at admission. Thereof, four false alerts were caused for each true alert and warnings were given in advance for $73 \%$ of all actual positive cases. The same model could be operationalised at a precision of 0.25 , which gave a warning for $13 \%$ of all episodes, resulting in three false alerts for each positive alert and a warning for $48 \%$ of all actual positive cases.

Just because we can predict future events does not mean we should [39]. Very few clinical prediction models have undergone formal impact analysis, i.e. studying the impact of using the predictions on patient 


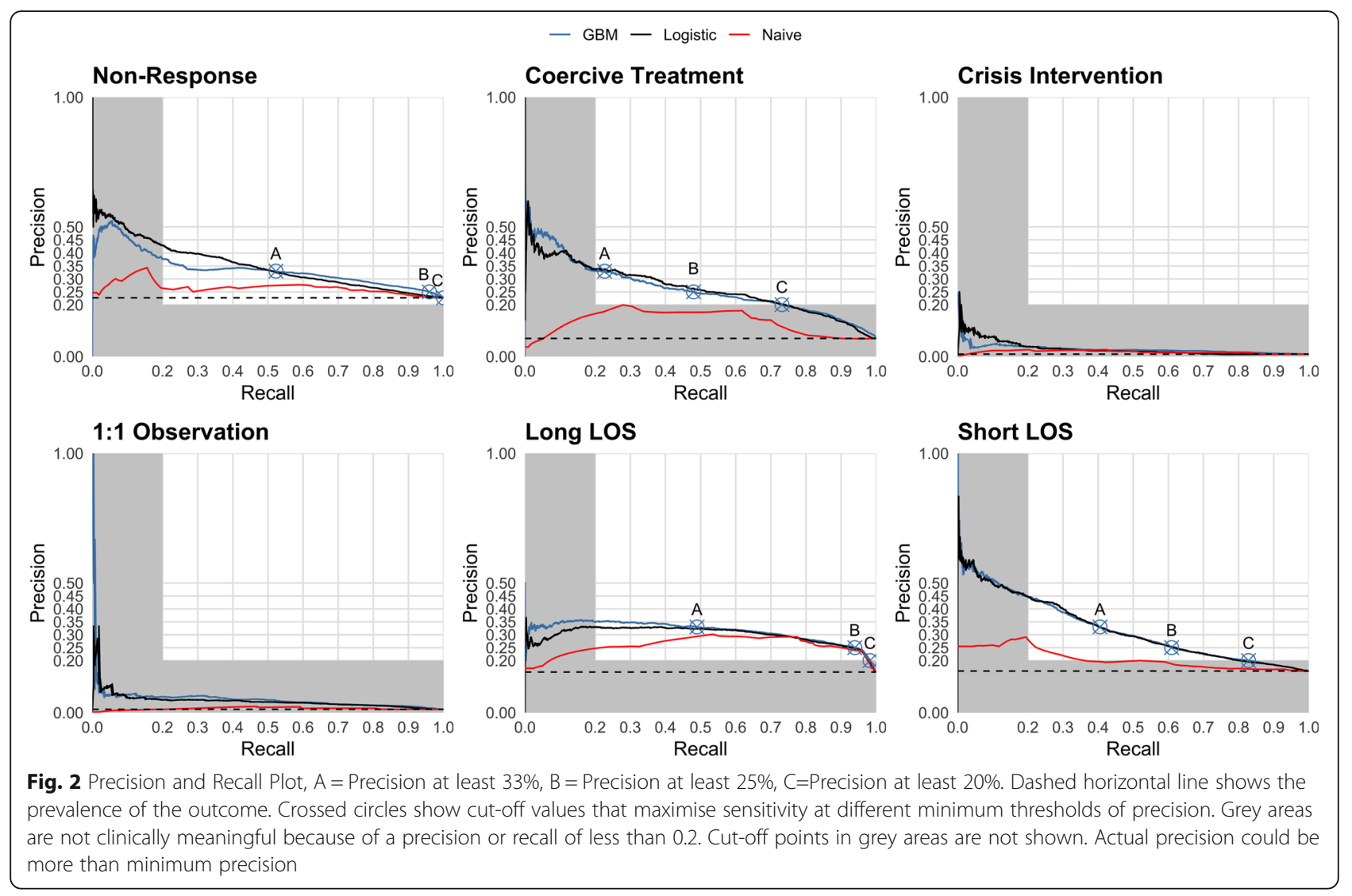

Table 2 Perfomance Measures

\begin{tabular}{|c|c|c|c|c|c|c|c|}
\hline & $\begin{array}{l}\text { Sensitivity / } \\
\text { Recall }\end{array}$ & Specificity & $\begin{array}{l}\text { Positive Predictive Value / } \\
\text { Precision }\end{array}$ & $\begin{array}{l}\text { Negative Predictive } \\
\text { Value }\end{array}$ & Prevalence & $\begin{array}{l}\text { Detection } \\
\text { Prevalence }\end{array}$ & $\begin{array}{l}\text { Balanced } \\
\text { Accuracy }\end{array}$ \\
\hline \multicolumn{8}{|c|}{ Precision at least $20 \%$} \\
\hline Non-Response & 1.00 & 0.00 & 0.23 & 1.00 & 0.23 & 1.00 & 0.50 \\
\hline $\begin{array}{l}\text { Coercive } \\
\text { Treatment }\end{array}$ & 0.73 & 0.78 & 0.20 & 0.97 & 0.07 & 0.26 & 0.76 \\
\hline Long LOS & 0.98 & 0.28 & 0.20 & 0.99 & 0.16 & 0.76 & 0.63 \\
\hline Short LOS & 0.83 & 0.37 & 0.20 & 0.92 & 0.16 & 0.66 & 0.60 \\
\hline \multicolumn{8}{|c|}{ Precision at least $25 \%$} \\
\hline Non-Response & 0.96 & 0.15 & 0.25 & 0.93 & 0.23 & 0.87 & 0.56 \\
\hline $\begin{array}{l}\text { Coercive } \\
\text { Treatment }\end{array}$ & 0.48 & 0.89 & 0.25 & 0.96 & 0.07 & 0.13 & 0.69 \\
\hline Long LOS & 0.94 & 0.48 & 0.25 & 0.98 & 0.16 & 0.58 & 0.71 \\
\hline Short LOS & 0.61 & 0.65 & 0.25 & 0.90 & 0.16 & 0.39 & 0.63 \\
\hline \multicolumn{8}{|c|}{ Precision at least 33\% } \\
\hline Non-Response & 0.52 & 0.69 & 0.33 & 0.83 & 0.23 & 0.36 & 0.61 \\
\hline $\begin{array}{l}\text { Coercive } \\
\text { Treatment }\end{array}$ & 0.23 & 0.97 & 0.33 & 0.94 & 0.07 & 0.05 & 0.60 \\
\hline Long LOS & 0.49 & 0.82 & 0.33 & 0.90 & 0.16 & 0.23 & 0.65 \\
\hline Short LOS & 0.41 & 0.84 & 0.33 & 0.88 & 0.16 & 0.20 & 0.62 \\
\hline
\end{tabular}

Outcomes without clinically meaningful operational points are not shown (Crisis Intervention \& 1:1 Observation). Actual precision could be more than minimum precision. TP True Positive, $F P$ False Positive, $T N$ True negative, $F N$ False Negative, Sensitivity $=T P /(T P+F N)$, Specificity $=T N /(T N+F P)$, Positive Predictive Value $=T P /$ $(T P+F P)$, Negative Predictive Value $=T N /(T N+F N)$, Prevalence $=(T P+F N) /(T P+F P+T N+F N)$, Detection Prevalence $=(T P+F P) /(T P+F P+T N+F N)$,

Balanced Accuracy $=($ Sensitivity + Specificity $) / 2$ 

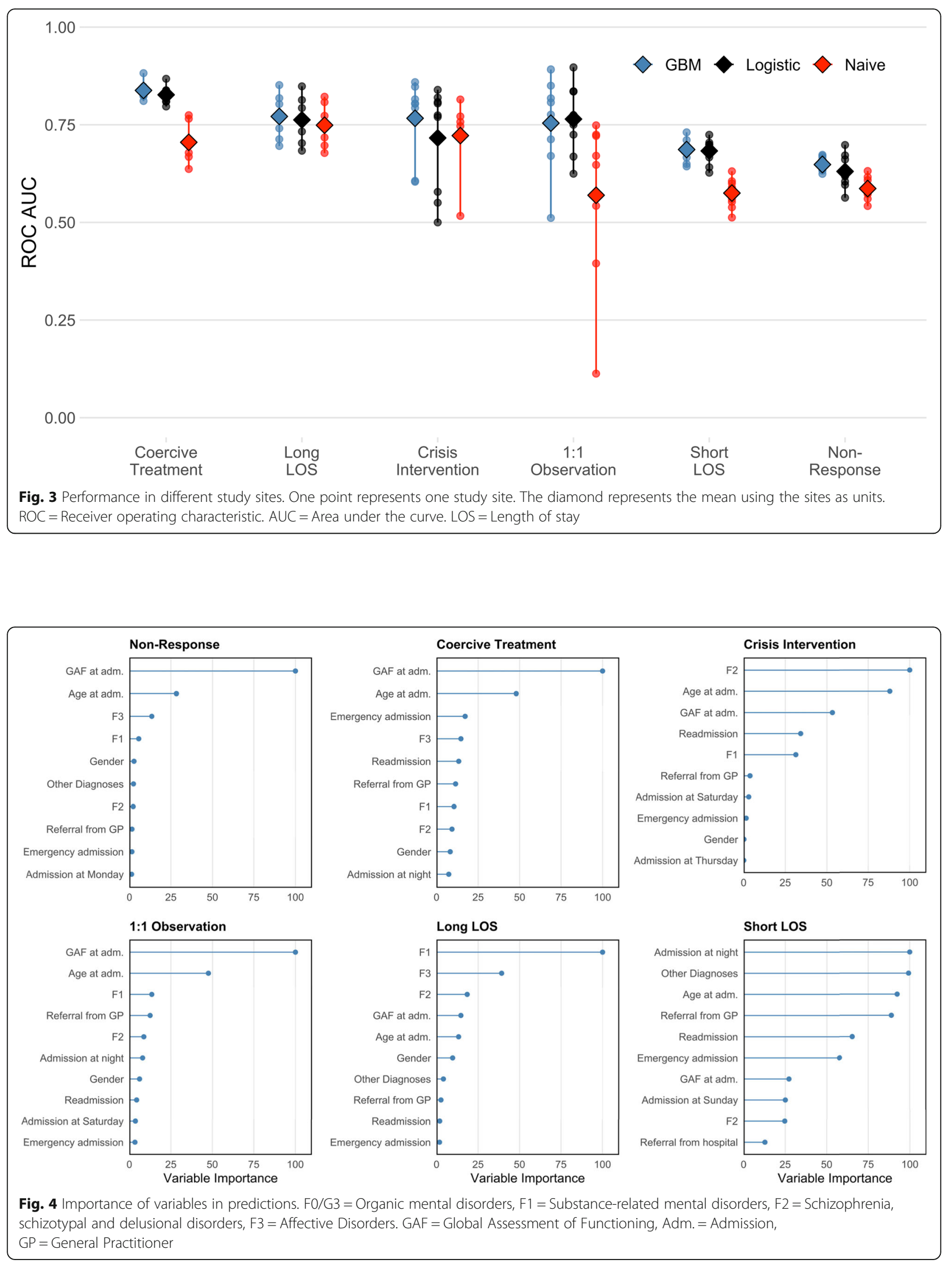
outcomes [40]. Patients' benefit depends on how predictions are translated into effective decision making [41]. Predictions must be reasonably included in the clinical processes to create an actual benefit from better informed decisions. This requires a range of steps at the individual hospital level, such as integration into current IT, human resources and financial investment systems.

Even a perfectly integrated model must be used responsibly in clinical practice, and the exact framework for such application is currently under a broad discussion [42-44]. For instance, caregivers have to be trained in using the provided results, patients'access to care has to remain equitable, real-world performance must be constantly scrutinised and responsibilities in case of errors have to be clear. Furthermore, predictions must not become selffulfilling. Instead, a warning at admission for coercive treatment could be used to intensify non-invasive care with the aim to avoid coercive approaches, for instance.

\section{Our study in comparison to previous research}

Less distinct diagnostic concepts [6-8], less standardization of care [9] and a broader spectrum of acceptable therapeutic regimes [10] make the prediction of outcomes in psychiatry more complex than in other medical disciplines [3-5]. An infamous example for these difficulties was the failure of the Medicare DRG system for psychiatry due to the inability to predict length of stay and associated hospital costs $[45,46]$. Recent studies have often used a broad range of feature variables in studies restricted to specific settings and patients. Leigthon et al. [47] predicted remission after 12 months in 79 patients with first episode of psychosis with a wide range of demographic, socioeconomic and psychometric feature variables and reached an area under the ROC of 0.65. Koutsouleris et al. [48] also investigated remission in first episode of psychosis and reached a sensitivity of $71 \%$, a specificity of $72 \%$ and a precision of $93 \%$ in 108 unseen patients with their top ten demographic, socioeconomic and psychometric predictor variables. Lin et al. [49] tried to distinguish treatment responders from nonresponders prior to antidepressant therapy in 455 patients with major depression. They used single nucleotide polymorphisms from genetic analyses and other clinical data and reached an area under the AUC of 0.82. Common traits of these studies were the restriction to specific patient groups and the relatively small sample sizes. Furthermore, they mainly used data that might not be available during routine patient admission.

\section{Strengths and weaknesses of our study}

A strength of this study was the large sample size over two distinct years and at nine study sites. This allowed us to include a broad range of the present spectrum of psychiatric inpatients and to develop models that should be applicable in most hospitals. Furthermore, we were able to test our models in patients that were treated in another calendar year and a different hospital and thereby reduce information leakage. A further strength of the present study was the restrictive inclusion of only feature variables that should be available at admission in most hospitals. Therefore, it should be possible to implement the present models in many hospitals without additional documentation effort.

A potential weakness of our study was the retrospective use of administrative routine data which entails potential validity concerns. The validity of routine hospital data for health services research is a frequently discussed topic [50, 51], and studies found both low [52] and high validity of such data [53]. However, the development of models for application in routine clinical practice necessitated the use of routinely generated data including the inherent caveats. A further limitation was the lack of time stamps for the diagnostic groupings. Patients were grouped in one of five basic diagnostic groups at admission and these groupings remained stable during an episode. However, we were not able to entirely rule out that these groupings might have been changed during the stay by staff in rare cases. A further limitation was the restriction to hospitals from one large provider of inpatient psychiatric services in the region of Hesse, Germany, which raises the question whether the predictive performance of our models would remain stable if applied in psychiatric hospitals with different circumstances.

\section{Conclusion}

The present study has shown that administrative routine data can be used to predict aspects relevant to the organisation of psychiatric hospital care. Such predictions could be applied to efficiently support hospital staff in their very own decision making and thereby increase quality of care. Future research should investigate the predictive performance that is necessary for a tool to be accepted by care givers and provide an effective assistance in the care process for the benefit of both staff and patients.

\section{Abbreviations \\ AUC: Area under the curve; GAF: Global Assessment of Functioning; \\ GBM: Gradient Boosting Machine; LOS: Length of stay; PR-Plots: Precision and} Recall plots; ROC: Receiver Operating Characteristic curve

\section{Acknowledgements \\ We would like to thank Philip Hehn for his support in editing the manuscript.}

\section{Authors' contributions \\ JW, AG, DJ, CN, KK, HB, KD, AK and MF conceived and designed the study. JW analysed and interpreted the data. AK initiated the research project. JW wrote the manuscript. All authors revised the manuscript critically for important intellectual content and approved the final version.}

\section{Funding}

This work was supported by the Innovation Fund of the Federal Joint Committee, Germany (grant number: 01VSF16009). The funding body played no role in the design of the study and collection, analysis, and interpretation of data and in writing the manuscript. 


\section{Availability of data and materials}

The datasets generated and/or analysed during the current study are not publicly available due confidentiality. The corresponding author will provide the script used for the statistical analyses upon reasonable request.

\section{Ethics approval and consent to participate}

This non-interventional, retrospective study used only de-identified patient data. No patient's personal identifying information is included in the manuscript. The study was approved by the ethics commission of the Medical Council Hesse, record number FF116/2017.

\section{Consent for publication}

Not applicable.

\section{Competing interests}

MF received funding from Janssen Cilag in 2014 for a project with children of parents with mental and behavioral disorders.

\section{Author details}

'Department of Psychiatry and Psychotherapy, Medical Center - University of Freiburg, Faculty of Medicine, University of Freiburg, Freiburg, Germany. ${ }^{2}$ Department of Business Development, Evangelical Foundation Neuerkerode, Braunschweig, Germany. ${ }^{3}$ Department of Business Development, Forensic Commitment and Quality Management, Vitos GmbH, Kassel, Germany. ${ }^{4}$ Vitos Hospital for Psychiatry und Psychotherapy, Kassel, Germany. Institute of Medical Biometry and Statistics, Medical Center - University of Freiburg, Faculty of Medicine, University of Freiburg, Breisgau, Germany. ${ }^{6}$ Vitos Hochtaunus, Friedrichsdorf, Germany. ${ }^{7}$ Heinrich-Heine-University, Düsseldorf, Germany. ${ }^{8}$ Vitos Hospital Giessen-Marburg, Giessen, Germany.

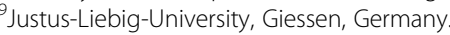

Received: 18 September 2019 Accepted: 31 January 2020 Published online: 06 February 2020

\section{References}

1. Amalberti $R$, Auroy $Y$, Berwick D, Barach P. Five system barriers to achieving ultrasafe health care. Ann Intern Med. 2005;142(9):756-64.

2. Ackermann G, Bergman MM, Heinzmann C, Läubli LM. Komplexitätsreduktion durch Klassifikationsmodelle in der Gesundheitsförderung und Prävention. In: Aspekte der Prävention Ausgewählte Beiträge des 3 Nationalen Präventionskongresses Dresden, 27 bis 28 November 2009. Stuttgart: Thieme; 2009. p. 20-9. Available from: http://edoc.unibas.ch/dok/A5254405.

3. Wolff J, McCrone P, Koeser L, Normann C, Patel A. Cost drivers of inpatient mental health care: a systematic review. Epidemiol Psychiatr Sci. 2015;24(01): 78-89.

4. Barry CL, Weiner JP, Lemke K, Busch SH. Risk adjustment in health insurance exchanges for individuals with mental illness. Am J Psychiatry. 2012;169(7): 704-9.

5. Montz E, Layton T, Busch AB, Ellis RP, Rose S, McGuire TG. Risk-adjustment simulation: plans may have incentives to distort mental health and substance use coverage. Health Aff Proj Hope. 2016;35(6):1022-8.

6. Wakefield JC. The concept of mental disorder: diagnostic implications of the harmful dysfunction analysis. World Psychiatry. 2007:6(3):149-56.

7. Aboraya A, Rankin E, France C, El-Missiry A, John C. The reliability of psychiatric diagnosis revisited. Psychiatry Edgmont. 2006;3(1):41-50.

8. Jablensky A. Psychiatric classifications: validity and utility. World Psychiatry. 2016:15(1):26-31.

9. Evans-Lacko SE, Jarrett M, McCrone $P$, Thornicroft G. Clinical pathways in psychiatry. Br J Psychiatry. 2008;193(1):4-5

10. Barbui C, Tansella M. Guideline implementation in mental health: current status and future goals. Epidemiol Psychiatr Sci. 2012;21(03):227-9.

11. Hastie T, Tibshirani R, Friedman J. The elements of statistical learning: data mining, inference, and prediction. 2nd ed. New York: Springer-Verlag; 2009

12. McKinney SM, Sieniek M, Godbole V, Godwin J, Antropova N, Ashrafian H, et al. International evaluation of an Al system for breast cancer screening. Nature. 2020;577(7788):89-94.

13. Tomašev N, Glorot X, Rae JW, Zielinski M, Askham H, Saraiva A, et al. A clinically applicable approach to continuous prediction of future acute kidney injury. Nature. 2019;572(7767):116-9.
14. Esteva A, Kuprel B, Novoa RA, Ko J, Swetter SM, Blau HM, et al. Dermatologist-level classification of skin cancer with deep neural networks. Nature. 2017;542(7639):115-8.

15. Litjens $\mathrm{G}$, Sánchez Cl, Timofeeva N, Hermsen M, Nagtegaal I, Kovacs I, et al. Deep learning as a tool for increased accuracy and efficiency of histopathological diagnosis. Sci Rep. 2016;6:26286.

16. Gulshan V, Peng L, Coram M, Stumpe MC, Wu D, Narayanaswamy A, et al. Development and validation of a deep learning algorithm for detection of diabetic retinopathy in retinal fundus photographs. JAMA. 2016;316(22):2402-10.

17. Schnyer DM, Clasen PC, Gonzalez C, Beevers CG. Evaluating the diagnostic utility of applying a machine learning algorithm to diffusion tensor MRI measures in individuals with major depressive disorder. Psychiatry Res Neuroimaging. 2017;264:1-9.

18. Berlyand Y, Raja AS, Dorner SC, Prabhakar AM, Sonis JD, Gottumukkala RV, et al. How artificial intelligence could transform emergency department operations. Am J Emerg Med. 2018;36(8):1515-7.

19. Hong WS, Haimovich AD, Taylor RA. Predicting hospital admission at emergency department triage using machine learning. PLoS One. 2018; 13(7):e0201016.

20. Jones SS, Thomas A, Evans RS, Welch SJ, Haug PJ, Snow GL. Forecasting daily patient volumes in the emergency department. Acad Emerg Med Off J Soc Acad Emerg Med. 2008;15(2):159-70.

21. Desautels T, Calvert J, Hoffman J, Jay M, Kerem Y, Shieh L, et al. Prediction of Sepsis in the intensive care unit with minimal electronic health record data: a machine learning approach. JMIR Med Inform. 2016;4(3):e28.

22. Horng S, Sontag DA, Halpern Y, Jernite Y, Shapiro NI, Nathanson LA Creating an automated trigger for sepsis clinical decision support at emergency department triage using machine learning. PLoS One. 2017; 12(4):e0174708.

23. Gultepe E, Green JP, Nguyen H, Adams J, Albertson T, Tagkopoulos I. From vital signs to clinical outcomes for patients with sepsis: a machine learning basis for a clinical decision support system. J Am Med Inform Assoc. 2014; 21(2):315-25.

24. Wolff J, McCrone P, Patel A, Normann C. Determinants of per diem hospital costs in mental health. PLoS One. 2016;11(3):e0152669.

25. Steyerberg EW, Moons KGM, van der Windt DA, Hayden JA, Perel P, Schroter $\mathrm{S}$, et al. Prognosis research strategy (PROGRESS) 3: prognostic model research. PLoS Med. 2013;10(2):e1001381.

26. Car J, Sheikh A, Wicks P, Williams MS. Beyond the hype of big data and artificial intelligence: building foundations for knowledge and wisdom. BMC Med. 2019;17(1):143

27. Ngiam KY, Khor IW. Big data and machine learning algorithms for healthcare delivery. Lancet Oncol. 2019;20(5):e262-73.

28. Tipping MD, Forth VE, Magill DB, Englert K, Williams MV. Systematic review of time studies evaluating physicians in the hospital setting. J Hosp Med Off Publ Soc Hosp Med. 2010;5(6):353-9.

29. Wolff J, Auber G, Schober T, Schwär F, Hoffmann K, Metzger M, et al. Worktime distribution of physicians at a German University hospital. Dtsch Arzteblatt Int. 2017:114(42):705-11.

30. Panch T, Mattie H, Celi LA. The "inconvenient truth" about Al in healthcare. Npj Digit Med. 2019;2(1):1-3.

31. Friedman $\mathrm{JH}$. Greedy function approximation: a gradient boosting machine. Ann Stat. 2001;29(5):1189-232.

32. Kuhn M. Building predictive models in R using the caret package. J Stat Softw. 2008:28(5):1-26.

33. R Core Team. R: A language and environment for statistical computing. Vienna: R Foundation for Statistical Computing; 2018. https://www.R-project.org/

34. Jones SH, Thornicroft G, Coffey M, Dunn G. A brief mental health outcome scale-reliability and validity of the global assessment of functioning (GAF). Br J Psychiatry. 1995;166(5):654-9.

35. DeLong ER, DeLong DM, Clarke-Pearson DL. Comparing the areas under two or more correlated receiver operating characteristic curves: a nonparametric approach. Biometrics. 1988;44(3):837-45

36. Saito T, Rehmsmeier $M$. The Precision-Recall Plot Is More Informative than the ROC Plot When Evaluating Binary Classifiers on Imbalanced Datasets. PLoS One. 2015;10(3):e0118432.

37. Emanuel EJ, Wachter RM. Artificial intelligence in health care: will the value match the hype? JAMA. 2019;321(23):2281-2.

38. Shah NH, Milstein A, Bagley SC. Making Machine Learning Models Clinically Useful. JAMA. 2019:322(14):1351-2. https://doi.org/10.1001/jama.2019.10306 Published online August 08. 
39. Vollmer S, Mateen BA, Bohner G, Király FJ, Ghani R, Jonsson P, et al. Machine learning and Al research for Patient Benefit: 20 Critical Questions on Transparency, Replicability, Ethics and Effectiveness. CoRR. 2018; abs/1812. 10404. Available from: http://arxiv.org/abs/1812.10404. Accessed 18 Sept 2019.

40. Reilly BM, Evans AT. Translating clinical research into clinical practice: impact of using prediction rules to make decisions. Ann Intern Med. 2006;144(3): 201.

41. Altman DG, Vergouwe $Y$, Royston P, Moons KGM. Prognosis and prognostic research: validating a prognostic model. BMJ. 2009;338:b605.

42. Char DS, Shah NH, Magnus D. Implementing machine learning in health care — addressing ethical challenges. N Engl J Med. 2018;378(11):981-3.

43. Vayena E, Blasimme A, Cohen IG. Machine learning in medicine: addressing ethical challenges. PLoS Med. 2018;15(11):e1002689.

44. Nebeker C, Torous J, Bartlett Ellis RJ. Building the case for actionable ethics in digital health research supported by artificial intelligence. BMC Med. 2019;17(1):137.

45. English JT, Sharfstein SS, Scherl DJ, Astrachan B, Muszynski IL. Diagnosisrelated groups and general hospital psychiatry: the APA study. Am J Psychiatry. 1986;143(2):131-9.

46. Wolff J, McCrone P, Patel A, Kaier K, Normann C. Predictors of length of stay in psychiatry: analyses of electronic medical records. BMC Psychiatry. 2015; 15(1):1.

47. Leighton SP, Krishnadas R, Chung K, Blair A, Brown S, Clark S, et al. Predicting one-year outcome in first episode psychosis using machine learning. PLoS One. 2019;14(3):e0212846.

48. Koutsouleris N, Kahn RS, Chekroud AM, Leucht S, Falkai P, Wobrock T, et al. Multisite prediction of 4-week and 52-week treatment outcomes in patients with first-episode psychosis: a machine learning approach. Lancet Psychiatry. 2016;3(10):935-46.

49. Lin E, Kuo P-H, Liu Y-L, Yu YW-Y, Yang AC, Tsai S-J. A deep learning approach for predicting antidepressant response in major depression using clinical and genetic biomarkers. Front Psychiatry. 2018;9:290.

50. Wolff J, Heister T, Normann C, Kaier K. Hospital costs associated with psychiatric comorbidities: a retrospective study. BMC Health Serv Res. 2018; 18(1):67.

51. Byrne N, Regan C, Howard L. Administrative registers in psychiatric research: a systematic review of validity studies. Acta Psychiatr Scand. 2005;112(6): 409-14.

52. Oiesvold T, Nivison M, Hansen V, Skre I, Ostensen L, Sørgaard KW. Diagnosing comorbidity in psychiatric hospital: challenging the validity of administrative registers. BMC Psychiatry. 2013;13:13.

53. Soo M, Robertson LM, Ali T, Clark LE, Fluck N, Johnston M, et al. Approaches to ascertaining comorbidity information: validation of routine hospital episode data with clinician-based case note review. BMC Res Notes. 2014;7: 253.

\section{Publisher's Note}

Springer Nature remains neutral with regard to jurisdictional claims in published maps and institutional affiliations.

Ready to submit your research? Choose BMC and benefit from:

- fast, convenient online submission

- thorough peer review by experienced researchers in your field

- rapid publication on acceptance

- support for research data, including large and complex data types

- gold Open Access which fosters wider collaboration and increased citations

- maximum visibility for your research: over $100 \mathrm{M}$ website views per year

At $\mathrm{BMC}$, research is always in progress.

Learn more biomedcentral.com/submissions 